\title{
Network Coding Techniques for Network Monitoring: a Brief Introduction.
}

\author{
Christina Fragouli \\ École Polytechnique Fédérale de Lausanne \\ Email: christina.fragouli@epfl.ch
}

\author{
Athina Markopoulou \\ University of California at Irvine \\ amarko@stanfordalumni.org
}

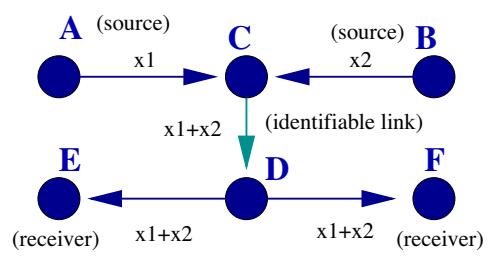

\begin{abstract}
Monitoring and diagnosis of network conditions is a central problem in networking. As such, it has received a lot of attention in the Internet community in general and in the context of overlay networks in particular. Independently, recent advances in network coding have shown that it is possible to increase network capacity and better share the available resources by allowing intermediate nodes to perform processing operations, in addition to just forwarding packets. We here review use of network coding techniques in this context. As a specific application, we examine the well-known problem of network tomography, and in particular for inferring link loss rates from end-to-end measurements. Use of network coding can decrease the bandwidth used by probes, improve the accuracy of estimation, and decrease the complexity of selecting paths or trees to send probes.
\end{abstract}

\section{INTRODUCTION}

In the past decade, active measurement techniques have been proposed that send sequences of probe packets from a set of sources to a set of receivers, and infer link-level metrics of interest from the received packets; in analogy to medical imaging this class of problems is commonly referred to as network tomography. For a detailed overview of related work please see [1], [2].

In this work, we propose the use of network coding techniques to improve several aspects of network monitoring. The basic idea of network coding is to allow intermediate nodes to process the incoming packets before forwarding them. The assumed capabilities of the nodes are realistic in the context of overlay networks, where the capabilities and operations of a node are completely controlled by the designer/operator of the overlay. This is in contrast to Internet routers, on which the end-user has no control. Furthermore, we envision using network coding only for measurement probes and not necessarily for the bulk of regular traffic.

\section{AN EXAMPLE}

The example network depicted in Fig. 1 illustrates our approach. Note that the multicast-based tomography approach would use two multicast trees rooted at nodes $A$ and $B$ and ending at $E$ and $F$, in order to cover all five links at least once. Our approach has the following advantages:

(i) The multicast trees approach would not distinguish the lossrates between links $A C$ and $C D$ (or similarly $B C$ and $C D$ ). (ii) In every experiment we send exactly one probe on every link, which is the minimum possible required to cover the
Fig. 1. An example configuration. $A$ and $B$ are sources that produce probe packets, $C$ adds (or xor-s) incoming packets, $D$ copies incoming packet to both outgoing links. The observations at receivers $E$ and $F$ are used to estimate the link loss rates.

entire graph. In contrast, the two multicast trees would overlap and send two probes on each one of the links $C D, D E$ and $D F$.

These observations gracefully generalize in arbitrary graphs.

\section{MAIN RESULTS}

Allowing nodes in an overlay network to perform network coding can improve all aspects of network tomography, namely bandwidth usage, estimation accuracy, and complexity in choosing which paths to monitor. More specifically, use of network coding techniques allows to:

(i) increase the number of links whose loss probability can be inferred from end-to-end measurements;

(ii) eliminate the overlap between paths and/or trees needed to cover the entire network;

(iii) use less probes per sequence to achieve a certain accuracy, by intelligently using not only the number, but also the content of received probes and

(iv) reduce the complexity of choosing which paths to monitor. We can formulate the minimum cost cover problem as a Linear Program (LP), which allows to solve it in polynomial time. This is an improvement over the same problem without network coding, which is NP-hard.

In general, we believe that the idea of combining network coding with network tomography techniques is very promising. Detailed discussion of the results, as well as simulation results, are provided in [1], [2].

\section{REFERENCES}

[1] C.Fragouli and A. Markopoulou, A network coding approach to network monitoring, Allerton 2005, Sept. 2005.

[2] C. Fragouli and A. Markopoulou, Network coding for network tomography, technical report in preparation. 
